\title{
Influence of periampullary diverticula on endoscopic retrograde cholangiopancreatography
}

\author{
HAILUN ZHENG, SHANJUN YAN, DAPENG LI, YONGJU XUE and XIAOJING DENG
}

Department of Gastroenterology, The First Affiliated Hospital of Bengbu Medical College, Bengbu, Anhui 233004, P.R. China

Received February 28, 2020; Accepted January 8, 2021

DOI: $10.3892 /$ etm.2021.9841

\begin{abstract}
The present study aimed to explore the influence of the presence of periampullary diverticula (PAD) on the implementation of endoscopic retrograde cholangiopancreatography (ERCP). A total of 388 patients with pancreaticobiliary disease who underwent ERCP for the first time between January 2017 and December 2018 were included and they were divided into a PAD group $(n=179)$ and non-PAD (N-PAD) group $(n=209)$ according to the presence or absence of PAD. A logistic regression model was used to analyze the risk factors for PAD. The prevalence of PAD in males was higher than that in females [odds ratio $(\mathrm{OR})=2.250,95 \% \mathrm{CI}$ : 1.670-3.801]. The prevalence of PAD in patients with bile duct stone was $57.92 \%$ and higher than that in patients without stone (OR=4.475, 95\% CI: 2.932-7.679). The morbidity of PAD in elderly patients with bile duct stone was higher than in those without stone (OR=6.728, 95\% CI: 3.790-11.943). Among the elderly patients, the constituent ratio of males in the PAD group was higher than that in the N-PAD group $\left(\chi^{2}=13.543\right.$, $\mathrm{P}<0.001)$. The constituent ratio of patients who underwent endoscopic sphincterotomy (EST) was lower than that in the N-PAD group $\left(\chi^{2}=10.800, \mathrm{P}<0.001\right)$. In conclusion, the occurrence of PAD was high in elderly males and closely related to the occurrence of bile duct stones.
\end{abstract}

\section{Introduction}

Periampullary diverticula (PAD) or peripapillary diverticula are saccular structures of the duodenal outpouchings, arising within a range of $2-3 \mathrm{~cm}$ around the ampulla of Vater (1). Relevant studies have reported that PAD are difficult to identify in patients aged $<40$ years and the prevalence of PAD increases with advancing age (2-5). Although the presence of PAD is usually asymptomatic, it may increase the occurrence of

Correspondence to: Dr Xiaojing Deng, Department of Gastroenterology, The First Affiliated Hospital of Bengbu Medical College, 287 Changhuai Road, Bengbu, Anhui 233004, P.R. China E-mail: xiaojingdeng123@163.com

Key words: endoscopic retrograde cholangiopancreatography, periampullary diverticula, elderly, bile duct stone diseases such as pancreatitis or choledocholithiasis according to the specific anatomical structure between PAD and the major duodenal papilla and pancreaticobiliary ampulla $(3,6)$. Age is considered to be a confounding factor, since the prevalence of diverticula and bile duct stones both increase along with age (7). Previous studies have demonstrated the association between PAD and the incidence of bile duct stones (8).

Endoscopic retrograde cholangiopancreatography (ERCP) is one of the preferred methods for the diagnosis and treatment of extrahepatic biliary and pancreatic diseases. With the popularity and development of ERCP, the detection rate of PAD has been markedly improved. The estimated occurrence of PAD in patients undergoing ERCP is $10-20 \%$ (9), and the prevalence and diameter of PAD increase with age (2-4). However, the influence of PAD on ERCP for the treatment of biliary and pancreatic diseases remains controversial. Certain studies have indicated that the presence of PAD does not increase the risk of failure of selective cannulation (2); however, other studies have demonstrated that the presence of PAD increases the difficulty associated with ERCP and the risk of complications $(8,10)$. Thus, further studies are required in order to fully determine the influence of PAD on ERCP.

In the present retrospective study, clinical and endoscopic data of patients with biliary and pancreatic diseases treated with ERCP over the past 2 years were collected and analyzed. The study was to evaluate the current situation of PAD and to examine the influence of the presence of PAD in elderly patients with bile duct stones who received ERCP treatment.

\section{Materials and methods}

Patients. The present single-center retrospective study was performed at the First Affiliated Hospital of Bengbu Medical College (Bengbu, China). The demographic data and details of the ERCP procedure in patients with pancreaticobiliary disease who underwent ERCP between January 2017 and December 2018 were obtained from the electronic ERCP database. Patients undergoing ERCP for the first time with complete information were included in the present study.

Patients who met the following criteria were excluded: i) History of choledochojejunostomy; ii) a fistula in the major duodenal papilla; iii) incomplete records of clinical and/or accessory examinations.

A total of 388 included cases were divided into the PAD group $(n=179)$ and the non-PAD (N-PAD) group $(n=209)$. 
Subjects aged $\geq 60$ years were defined as elderly (11). All patients with complete information from hospital records on general data, laboratory and imaging examination were used to determine the initial diagnosis and ERCP indications, and contraindications were excluded. This study was approved by the Ethics Committee of the First Affiliated Hospital of Bengbu Medical College (Bengbu, China; approval no. 2019KY030).

Collection of baseline information. Baseline information, including sex, age and diseases, was first retrieved for the patients of the present study. Subsequently, the characteristics including age distribution were analyzed in patients with cholangiolithiasis.

Procedures.AllERCP procedures were performedorsupervised by qualified physicians with $>10$ years of experience in ERCP. The duodenoscope (Olympus JF-260/TJF-260), CleverCut sphincterotome (Olympus Medical Systems Co. Ltd.), guidewire, dilation balloon, extraction balloon, stone extractor, nasobiliary drainage, various types of biliopancreatic stents and related instruments were applied under X-ray fluoroscopy. The operators and patients received standard radiation protection. Patients received oral dimethicone $30 \mathrm{~min}$ prior to surgery and an intramuscular injection of anisodamine, meperidine and/or diazepam (depending on the age and general condition) 15 min prior to surgery for non-anesthetic sedation and analgesia pretreatment.

The position of the papilla was determined after the duodenoscope was moved to the descending part of the duodenum. The shape, opening and the presence of PAD were observed. The type, quantity and long diameter of PAD were recorded in patients with PAD. Conventional selective cannulation was performed using a cutting guide wire. Procedures including pancreatic guide wire-assisted biliary cannulation, precut sphincterotomy, needle-knife fistulosphincterotomy and clip-assisted cannulation were performed when conventional intubation failed. After the guide wire entered the biliary duct, the biliopancreatic duct lesion and its position were confirmed according to the condition of contrast agent under X-ray fluoroscopy. The methods of opening and lithotomy included endoscopic sphincterotomy (EST), endoscopic papillary balloon dilatation (EPBD) and EST+EPBD. One of the three options was performed according to the number and the diameter of stones, and the position and the side bulge length of the papilla. After stone removal, the bile duct was examined by extraction balloon cholangiography to confirm the absence of residual stones. After the stone was removed, the operation of washing by noradrenaline in iced saline solutions, balloon compression and hemostasis using a metal clip was performed according to the papilla bleeding. The biliary stent was simply placed when stones were not able to be removed at one time. The types of PAD and the application of EPBD in PAD patients with bile duct stones are shown in Fig. 1. Of which, Fig. 1A is type I PAD (the duodenal papilla is located inside the diverticulum); Fig. 1B is type II PAD (the duodenal papilla lies at the margin of the diverticulum); Fig. $1 \mathrm{C}$ is type III PAD (the duodenal papilla is located near the diverticulum); Fig. 1D shows dilated sphincter using EPBD for type I PAD patients with bile duct stones; Fig. 1E shows the stone removal after EPBD treatment; and Fig. 1F shows that EPBD dilates the sphincter under the X-ray fluoroscopy.
Diagnostic criteria. The diameter of the depressed intestinal wall, which was $>5 \mathrm{~mm}$ within $2-3 \mathrm{~cm}$ of the main nipple on the descending side of the duodenum, was defined as the PAD. The size and quantity of the diverticula were confirmed in relation to the scale of computed tomography and magnetic resonance cholangiopancreatography (MRCP) examination images combined with direct view images acquired with the endoscope with reference to the dilated balloon. A diverticulum with a length $\geq 3 \mathrm{~cm}$ was defined as a giant diverticulum, while a quantity $\geq 2$ was defined as multiple diverticula (12).

Difficult biliary cannulation refers to the failure of conventional instruments and methods to enter the bile duct ( $>5$ contacts with the papilla whilst attempting to cannulate or $>5$ min spent attempting to cannulate following visualization of the papilla or $>1$ unintended pancreatic duct cannulation or opacification) (13).

The criteria for sphincter of Oddi dysfunction (SOD) were as follows (14): i) Typical, episodic biliary or pancreatic pain; ii) $>1.5$ - to 2.0 -fold elevation in liver-associated enzymes (aminotransferases or alkaline phosphatase levels) or pancreatic enzymes (amylase or lipase) on at least 2 occasions during episodes of pain; iii) MRCP and ERCP revealed dilated common bile duct (bile duct diameter $\geq 10 \mathrm{~mm}$ ) or main pancreatic ducts (pancreatic duct diameter $\geq 6 \mathrm{~mm}$ in the head and $\geq 5 \mathrm{~mm}$ in the body), and excluded other biliary and pancreatic diseases; iv) duodenal papillary sclerosis resulted in difficult dilatation and required high-pressure balloon sphincter stretching; v) following EST or sphincter stretching, the clinical symptoms improved significantly.

The definition of large balloon dilation is that the dilation of the columnar balloon in the papillary sphincter is $\geq 12 \mathrm{~mm}$ (15). ERCP-related adverse reactions, including pancreatitis, cholangitis, hemorrhage and perforation, were defined according to the American Society for Gastrointestinal Endoscopy guidelines (16).

Statistical analysis. SAS 9.4 software (SAS Institute Inc.) was used for statistical analysis. Count data are expressed as n(\%) and the chi-squared test was used to analyze these data. Data with a normal distribution are expressed as the mean \pm standard deviation and the Student's t-test was adopted for the comparison of these data between groups. Univariate and multivariate logistic regression analyses were used to assess the influencing factors of PAD. $\mathrm{P}<0.05$ was considered to indicate a statistically significant difference.

Sample size calculation was performed according to the following formula:

$$
\mathrm{N}=\frac{2\left(Z_{\frac{\alpha}{2}}+Z_{\beta}\right)^{2}(\overline{p q})}{\left(P_{0}-P_{1}\right)^{2}}
$$

where $\mathrm{P}_{0}$ is the incidence of bile duct stones in the N-PAD group and $\mathrm{P}_{1}$ is the incidence of bile duct stones in the PAD group. $\mathrm{Q}$ is the mean value of $\left(1-\mathrm{P}_{0}\right)$ and $\left(1-\mathrm{P}_{1}\right)$. The sample proportion of the two groups was $1: 1$ and the total sample size was $n=2 N$. In a previous study (17), $P_{1}=0.47$ and $P_{0}=0.16$ had been calculated and the required sample for each group was 70 . The sample size in the present study was in accordance with this. 
Table I. The characteristics of cases who received endoscopic retrograde cholangiopancreatography treatment between the two groups.

\begin{tabular}{|c|c|c|c|c|c|}
\hline Variables & Total & N-PAD & PAD & Statistics & P-value \\
\hline Age, mean \pm SD & $67.60 \pm 14.71$ & $63.91 \pm 15.58$ & $71.91 \pm 12.34$ & $t=-5.63$ & $<0.001$ \\
\hline Sex, n $(\%)$ & & & & $\chi^{2}=19.766$ & $<0.001$ \\
\hline Male & $202(52.06)$ & $87(41.63)$ & $115(64.25)$ & & \\
\hline Female & $186(47.94)$ & $122(58.37)$ & $64(35.75)$ & & \\
\hline Disease, n (\%) & & & & $\chi^{2}=24.002$ & 0.001 \\
\hline Pancreatic diseases & $5(1.29)$ & $5(2.39)$ & $0(0.00)$ & & \\
\hline SOD & $13(3.35)$ & $8(3.83)$ & $5(2.79)$ & & \\
\hline Ampullary and papillary tumors & $15(3.87)$ & $14(6.70)$ & $1(0.56)$ & & \\
\hline Malignant bile duct stricture & $85(21.91)$ & $65(31.10)$ & $20(11.17)$ & & \\
\hline Bile duct stones combined with cholecystolithiasis & $17(4.38)$ & $7(3.35)$ & $10(5.59)$ & & \\
\hline Bile duct stones combined with acute cholangitis & $26(6.70)$ & $11(5.26)$ & $15(8.38)$ & & \\
\hline Benign bile duct stricture & $11(2.84)$ & $8(3.83)$ & $3(1.68)$ & & \\
\hline Bile duct stones & $216(55.67)$ & $91(43.54)$ & $125(69.83)$ & & \\
\hline
\end{tabular}

SOD, sphincter of Oddi dysfunction; PAD, periampullary diverticula.

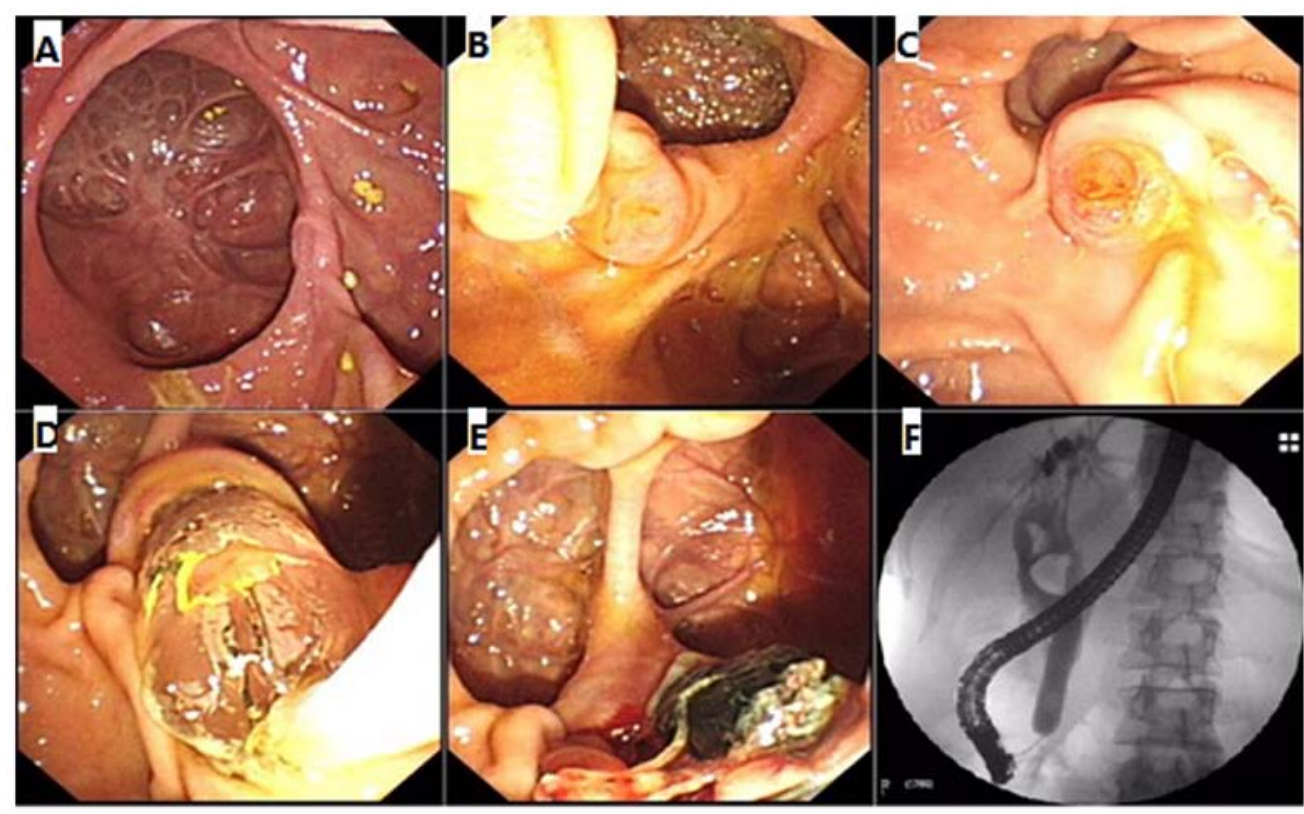

Figure 1. Papilla opening and lithotomy. (A) Type I PAD; (B) type II PAD; (C) type III PAD; (D) dilated sphincter by EPLBD; (E) stone removal; (F) EPLBD dilates the sphincter under the X-ray fluoroscopy. EPLBD, endoscopic papillary large balloon dilation; PAD, periampullary diverticula.

\section{Results}

Patients. A total of 388 patients with complete information who underwent ERCP for the first time were included, with a mean age of $67.60 \pm 14.71$ years (range, 36-93 years), and the male to female ratio was 1.09. The mean age of the 179 patients in the PAD group was $71.91 \pm 12.34$ years and that of the 209 cases in the N-PAD group was $63.91 \pm 15.58$ years. There were statistical differences in age and sex between the PAD and N-PAD groups (Table I).

Prevalence of PAD in patients. In the present study, the prevalence of PAD was $56.93 \%(n=115)$ in males, $58.08 \%(n=150)$ in subjects with age $\geq 60$ years and $57.87 \%(n=125)$ in cases with bile duct stones, respectively.

Univariate regression analysis for PAD. As shown in Table II, the differences were discovered regarding male $(\mathrm{OR}=2.250$, 95\% CI: $1.670-3.801)$, age $\geq 60$ years $(\mathrm{OR}=3.021,95 \% \mathrm{CI}$ : 1.855-4.902), ampullary and papillary tumors $(\mathrm{OR}=0.052$, 95\% CI: 0.007-0.403) between the PAD and N-PAD groups.

Multivariate binary logistic regression analysis for PAD. The results of the multivariate binary logistic regression indicated that the prevalence of PAD in males was 2.519-fold that in females (OR=2.519, 95\% CI: 1.586-3.999). The risk of PAD 
Table II. Univariate and multivariate binary logistic regression analysis for PAD.

\begin{tabular}{lccr}
\hline Variables & OR $(95 \% \mathrm{CI})$ & OR $(95 \% \mathrm{CI})$ & P-value \\
\hline Sex & & & $<0.001$ \\
Male & $2.250(1.670-3.801)$ & $2.519(1.586-3.999)$ & \\
Female & Reference & Reference & \\
Age, years & Reference & & \\
$<60$ & Reference & $<0.001$ \\
$\geq 60$ & $3.021(1.855-4.902)$ & $3.066(1.798-5.228)$ & \\
Disease & & & 0.605 \\
Pancreatic diseases & - & - & 0.006 \\
SOD & $0.455(0.144-1.436)$ & $0.727(0.217-2.433)$ & $<0.001$ \\
Ampullary and papillary tumors & $0.052(0.007-0.403)$ & $0.054(0.007-0.429)$ & 0.751 \\
Malignant bile duct stricture & $0.224(0.127-0.396)$ & $0.181(0.099-0.330)$ & 0.719 \\
Bile duct stones combined with cholecystolithiasis & $1.040(0.381-2.835)$ & $0.841(0.290-2.442)$ & 0.006 \\
Bile duct stones combined with acute cholangitis & $0.993(0.436-2.262)$ & $1.176(0.486-2.842)$ & \\
Benign bile duct stricture & $0.273(0.070-1.057)$ & $0.380(0.091-1.591)$ & \\
Bile duct stones & Reference & Reference & \\
\hline
\end{tabular}

SOD, sphincter of Oddi dysfunction; OR, odds ratio.

among the elderly was 3.066-fold that of younger patients (OR=3.066, 95\% CI: 1.798-5.228). The occurrence of PAD in patients with malignant bile duct strictures was 0.181 -fold that of bile duct stones (OR=0.181, 95\% CI: 0.099-0.330; Table II).

Risk factors of PAD in elderly patients with bile duct stones. Among elderly patients, the constituent ratio of males in the PAD group was significantly higher than that in the N-PAD group $\left(\chi^{2}=13.543, \mathrm{P}<0.001\right)$ and the constituent ratio of patients undergoing EST in the PAD group was lower than that in the N-PAD group $\left(\chi^{2}=10.800, \mathrm{P}<0.001\right)$. No statistically significant differences in papilla cannulation, maximum diameter of stone, multiplicity/property of stones, lithotomy status, mechanical lithotripsy, complications, common bile duct diameter and repeated intervention were observed between the two groups $(\mathrm{P}>0.05$; Table III).

\section{Discussion}

PAD have gradually received increasing attention due to their specific location. With the in-depth development of ERCP, the detection rate of PAD has been greatly increased (9). The present study was performed to explore the current situation of PAD and to analyze the influence of the presence of PAD on the implementation of ERCP. The results confirmed that the prevalence of PAD in elderly patients (age, $\geq 60$ years) was higher, indicating that the presence of PAD is significantly associated with age. The age-prone prevalence of PAD may be related to the deterioration of the muscular wall and the intestinal wall, the weakening of intestinal tone contraction, the slowing of peristalsis and the abnormal retention of intestinal contents in the elderly (5). To date, PAD has been insufficiently studied in elderly populations treated with ERCP. Regarding PAD in elderly patients with bile duct stones, univariate analysis and multifactorial analysis were performed in the present study in order to further clarify the prevalence of PAD and the impact of PAD on ERCP.

The present study also indicated an association between the occurrence of PAD and sex, as the prevalence of PAD in males was higher than that in females, irrespective of the age of the patients. To the best of our knowledge, the present study was the first to determine that female sex may be a protective factor against PAD. Compared with that in patients with bile duct stones, the occurrence of PAD in patients with ampullary and papillary tumors and malignant bile duct stricture was lower. The prevalence of PAD in patients with bile duct stones was $57.92 \%$ and higher than in those without stone. The present results suggested that PAD are closely associated with the occurrence of bile duct stones and Sun et al (18) and Hall et al (19) also confirmed the relationship between PAD and bile duct stones. In the current study, analysis of patients with or without bile duct stones indicated that the difference in age between the PAD and the N-PAD group was mainly reflected in the population of bile duct stones and patients with bile duct stones exhibited a higher occurrence of PAD than other pancreatic biliary diseases.

The high prevalence of bile duct stones in patients with PAD may be due to the following: PAD causes SOD, resulting in inadequate drainage or regurgitation of bile and pancreatic juice; food deposition in the diverticulum easily occurs secondary to diverticulitis, causing constrictive papillitis, affecting the discharge of biliary sludge and increasing the incidence of retrograde biliary infection; exogenous glucuronidase increases the formation of free bilirubin, which combines with calcium salts to form the core of the stone and the $\mathrm{pH}$ value of the bile duct decreases when the bile duct is infected, which may promote the formation of pigment gallstones (20). Therefore, patients with bile duct stones aged $\geq 60$ years were selected as the key research subjects in the present study in order to explore the effects of the presence of PAD on ERCP. 
Table III. Effects of PAD in elderly patients with bile duct stones who received ERCP.

\begin{tabular}{|c|c|c|c|c|c|}
\hline \multirow[b]{2}{*}{ Variates } & \multirow[b]{2}{*}{ Total } & \multicolumn{2}{|c|}{ Group, n (\%) } & \multirow[b]{2}{*}{$\chi^{2}$} & \multirow[b]{2}{*}{ P-value } \\
\hline & & PAD & N-PAD & & \\
\hline Sex & & & & 13.543 & $<0.001$ \\
\hline Male & 115 & $89(68.99)$ & $26(41.27)$ & & \\
\hline Female & 77 & $40(31.01)$ & $37(58.73)$ & & \\
\hline Papilla cannulation & & & & 2.694 & 0.101 \\
\hline Difficult cannulation & 30 & $16(12.90)$ & $14(22.22)$ & & \\
\hline Routine cannulation & 157 & $108(87.10)$ & $49(77.78)$ & & \\
\hline Papilla cutting & & & & 10.800 & 0.001 \\
\hline Simple sphincter stretching & 49 & $42(33.33)$ & $7(11.11)$ & & \\
\hline EST & 140 & $84(66.67)$ & $56(88.89)$ & & \\
\hline Maximum diameter of stone, $\mathrm{cm}$ & & & & 4.240 & 0.120 \\
\hline $1.0-1.9$ & 112 & $79(65.83)$ & $33(60.00)$ & & \\
\hline$<1.0$ & 42 & $24(20.00)$ & $18(32.73)$ & & \\
\hline$\geq 2$ & 21 & $17(14.17)$ & $4(7.27)$ & & \\
\hline Multiplicity/property of stones & & & & 1.359 & 0.507 \\
\hline Single & 68 & $43(34.13)$ & $25(39.68)$ & & \\
\hline Multiple & 101 & $71(56.35)$ & $30(47.62)$ & & \\
\hline Mud-like and not formed & 20 & $12(9.52)$ & $8(12.70)$ & & \\
\hline Lithotomy status & & & & - & 0.648 \\
\hline Partial stone extraction + biliary stent & 10 & $8(6.40)$ & $2(3.17)$ & & \\
\hline Complete stone extraction & 168 & $111(88.80)$ & $57(90.48)$ & & \\
\hline Biliary stent inserted without stone extraction & 10 & $6(4.80)$ & $4(6.35)$ & & \\
\hline Mechanical lithotripsy & & & & 0.619 & 0.432 \\
\hline Unsuccessful & 166 & $109(86.51)$ & $57(90.48)$ & & \\
\hline Successful & 23 & $17(13.49)$ & $6(9.52)$ & & \\
\hline Complications & & & & - & 0.162 \\
\hline Hemorrhage & 1 & $0(0.00)$ & $1(1.59)$ & & \\
\hline Cholangitis & 153 & $106(82.81)$ & $47(74.60)$ & & \\
\hline Pancreatitis & 37 & $22(17.19)$ & $15(23.81)$ & & \\
\hline Common bile duct diameter, $\mathrm{cm}$ & & & & 0.653 & 0.419 \\
\hline$<1.50$ & 63 & $40(31.75)$ & $23(37.70)$ & & \\
\hline$\geq 1.5$ & 124 & $86(68.25)$ & $38(62.30)$ & & \\
\hline Repeated intervention & & & & - & 0.150 \\
\hline No & 177 & $116(89.92)$ & $61(96.83)$ & & \\
\hline Yes & 15 & $13(10.08)$ & $2(3.17)$ & & \\
\hline
\end{tabular}

PAD, periampullary duodenal diverticula; N-PAD group, patients without PAD; EST, endoscopic sphincterotomy

Selective cannulation of the bile duct through the main papilla is the primary method for ERCP lithotomy and the time and the route of cannulation influence the risk of post-ERCP pancreatitis (21). PAD has been reported to increase the difficulty and operating time of deep cannulation (19). However, the presence of PAD has also been determined to improve the success rate of intubation and to be an independent factor for easier cannulation (22). There was no significant effect on selective bile duct cannulation, even with stricter requirements and time limits for ERCP in elderly patients, and these results are supported by previous studies $(22,23)$. In the present study, multivariate binary logistic regression analysis revealed that the risk of PAD in males was 2.519 times that of females among the elderly. The means of papilla cutting were different in elderly patients with bile duct stones. The prevalence of elderly patients aged $\geq 60$ years who were subjected to simple sphincter stretching was 4.593 times that of those who underwent EST. This result suggested that the presence of PAD may be associated with a decreased usage rate of EST.

Considering the difficulty associated with ERCP, the present study provided suggestions which may be used to circumvent the difficulties associated with ERCP and the following steps may be suggested: i) Careful and complete examination prior to ERCP; ii) risk assessment and implementation of preventive measures for complications prior to ERCP; iii) comprehensive evaluation of indications for ERCP with a strict control of 
indications; iv) standardization of ERCP surgery; v) adequate preparation of ERCP operating consumables and instruments.

There are certain limitations to the present study that require to be mentioned: i) this was a retrospective single center study; ii) the conservative attitude of the physicians regarding subjective sphincter incision in patients with PAD. A larger sample size and a well-designed prospective randomized controlled trial are required to further confirm the results of the present study.

In conclusion, the present study indicated that the prevalence of patients with PAD was high among the elderly who received ERCP treatment, males exhibited a higher prevalence of PAD than females.

\section{Acknowledgements}

Not applicable.

\section{Funding}

This study was supported by the Key Research Project of Anhui Provincial University Natural Science Fund (grant no. KJ2019A0336) and the Anhui Provincial University Natural Science Fund Project (grant no. 1808085MH240).

\section{Availability of data and materials}

The datasets used and/or analyzed during the current study are available from the corresponding author on reasonable request.

\section{Authors' contributions}

HZ and XD designed the study. HZ wrote the manuscript. SY and YX contributed to data collection. HZ and DL analyzed and interpreted the data. YX contributed to the literature search. XD critically reviewed the manuscript for important intellectual content. HZ, SY, YX and DL confirmed the authenticity of all the raw data. All authors read and approved the final manuscript.

\section{Ethics approval and consent to participate}

The research protocol was examined and approved by the Ethics Committee of the First Affiliated Hospital of Bengbu Medical College (approval no. 2019KY030).

\section{Patient consent for publication}

Not applicable.

\section{Competing interests}

The authors declare that they have no competing interests.

\section{References}

1. Lobo DN, Balfour TW, Iftikhar SY and Rowlands BJ: Periampullary diverticula and pancreaticobiliary disease. Br J Surg 86: 588-597, 1999.

2. Zippi M, Traversa G, Pica R, De Felici I, Cassieri C, Marzano C, Occhigrossi $\mathrm{G}$ and Paoluzi P: Efficacy and safety of endoscopic retrograde cholangiopancreatography (ERCP) performed in patients with Periampullary duodenal diverticula (PAD). Clin Ter 165: e291-e294, 2014.
3. Chen L, Xia L, Lu Y, Bie L and Gong B: Influence of periampullary diverticulum on the occurrence of pancreaticobiliary diseases and outcomes of endoscopic retrograde cholangiopancreatography. Eur J Gastroenterol Hepatol 29: 105-111, 2017.

4. Örmeci N, Deda X, Kalkan Ç, Tüzün AE, Karakaya F, Dökmeci A, Bahar DK, Özkan H, Ídilman R and Çınar K: Impact of periampullary diverticula on bile duct stones and ampullary carcinoma. Euroasian J Hepatogastroenterol 6: 31-34, 2016.

5. Tyagi P, Sharma P, Sharma BC and Puri AS: Periampullary diverticula and technical success of endoscopic retrograde cholangiopancreatography. Surg Endosc 23: 1342-1345, 2009.

6. Li X, Zhu K, Zhang L, Meng W, Zhou W, Zhu X and Li B: Periampullary diverticulum may be an important factor for the occurrence and recurrence of bile duct stones. World J Surg 36: 2666-2669, 2012.

7. Neoptolemos JP: Endoscopic sphincterotomy in acute gallstone pancreatitis. Br J Surg 80: 547-549, 1993.

8. Kim KH and Kim TN: Endoscopic papillary large balloon dilation in patients with periampullary diverticula. World J Gastroenterol 19: 7168-7176, 2013.

9. Egawa N, Anjiki H, Takuma K and Kamisawa T: Juxtapapillary duodenal diverticula and pancreatobiliary disease. Dig Surg 27: 105-109, 2010.

10. Karaahmet F and Kekilli M: The presence of periampullary diverticulum increased the complications of endoscopic retrograde cholangiopancreatography. Eur J Gastroenterol Hepatol 30: 1009-1012, 2018.

11. Liang K: Differential associations between subjective age and depressive symptoms among urban and rural Chinese older adults. Aging Ment Health 24: 1271-1277, 2020.

12. Kim CW, Chang JH, Kim JH, Kim TH, Lee IS and Han SW: Size and type of periampullary duodenal diverticula are associated with bile duct diameter and recurrence of bile duct stones. J Gastroenterol Hepatol 28: 893-898, 2013.

13. Testoni PA, Mariani A, Aabakken L, Arvanitakis M, Bories E, Costamagna G, Devière J, Dinis-Ribeiro M, Dumonceau JM, Giovannini M, et al: Papillary cannulation and sphincterotomy techniques at ERCP: European Society of Gastrointestinal Endoscopy (ESGE) Clinical Guideline. Endoscopy 48: 657-683, 2016.

14. Nakeeb A: Sphincter of Oddi dysfunction: How is it diagnosed? How is it classified? How do we treat it medically, endoscopically, and surgically? J Gastrointest Surg 17: 1557-1558, 2013.

15. Zulli C, Grande G, Tontini GE, Labianca O, Geraci G, Sciumè C Antypas P, Fiocca F, Manes G, Devani M, et al: Endoscopic papillary large balloon dilation in patients with large biliary stones and periampullary diverticula: Results of a multicentric series. Dig Liver Dis 50: 828-832, 2018.

16. Chandrasekhara V, Khashab MA, Muthusamy VR, Acosta RD, Agrawal D, Bruining DH, Eloubeidi MA, Fanelli RD, Faulx AL, Gurudu SR, et al; ASGE Standards of Practice Committee: Adverse events associated with ERCP. Gastrointest Endosc 85: 32-47, 2017.

17. Tazuma S, Unno M, Igarashi Y, Inui K, Uchiyama K, Kai M, Tsuyuguchi $\mathrm{T}$, Maguchi $\mathrm{H}$, Mori $\mathrm{T}$, Yamaguchi $\mathrm{K}$, et al: Evidence-based clinical practice guidelines for cholelithiasis 2016. J Gastroenterol 52: 276-300, 2017.

18. Sun Z, Bo W, Jiang P and Sun Q: Different types of periampullary duodenal diverticula are associated with occurrence and recurrence of bile duct stones: A case-control study from a Chinese center. Gastroenterol Res Pract 2016: 9381759, 2016.

19. Hall RI, Ingoldby CJ and Denyer ME: Periampullary diverticula predispose to primary rather than secondary stones in the common bile duct. Endoscopy 22: 127-128, 1990.

20. Miyazaki S, Sakamoto T, Miyata M, Yamasaki Y, Yamasaki H and Kuwata K: Function of the sphincter of Oddi in patients with juxtapapillary duodenal diverticula: Evaluation by intraoperative biliary manometry under a duodenal pressure load. World J Surg 19: 307-312, 1995.

21. Lawrence C, Romagnuolo J, Cotton PB, Payne KM and Hawes RH: Post-ERCP pancreatitis rates do not differ between needle-knife and pull-type pancreatic sphincterotomy techniques: A multiendoscopist 13-year experience. Gastrointest Endosc 69: 1271-1275, 2009.

22. Panteris V, Vezakis A, Filippou G, Filippou D, Karamanolis D and Rizos S: Influence of juxtapapillary diverticula on the success or difficulty of cannulation and complication rate. Gastrointest Endosc 68: 903-910, 2008.

23. Tham TC and Kelly M: Association of periampullary duodenal diverticula with bile duct stones and with technical success of endoscopic retrograde cholangiopancreatography. Endoscopy 36: 1050-1053, 2004. 